\title{
Molecular diversity analysis of genotypes from four Aegilops species based on retrotransposon-microsatellite amplified polymorphism (REMAP) markers
}

\author{
Justyna Leśniowska-Nowak ${ }^{1}$ (D) Sylwia Okon ${ }^{1}\left[\right.$. Aleksandra Wieremczuk ${ }^{1}$
}

Received: 13 February 2020 / Accepted: 9 September 2020 / Published online: 21 September 2020

(c) The Author(s) 2020

\begin{abstract}
Genetic diversity analysis is an important tool in crop improvement. Species with high genetic diversity are a valuable source of variation used in breeding programs. The aim of this study was to assess the genetic diversity of four species belonging to the genus Aegilops, which are often used to expand the genetic variability of wheat and triticale. Forty-five genotypes belonging to the genus Aegilops were investigated. Within- and among-species genetic diversity was calculated based on REMAP (retrotransposon-microsatellite amplified polymorphism) molecular markers. Obtained results showed that REMAP markers are a powerful method for genetic diversity analysis, which produces a high number of polymorphic bands $(96.09 \%$ of total bands were polymorphic). Among tested genotypes, Ae. crassa and Ae. vavilovii showed the highest genetic diversity and should be chosen as a valuable source of genetic variation.
\end{abstract}

Keywords Aegilops $\cdot$ Genetic diversity $\cdot$ Molecular markers $\cdot$ REMAP

\section{Introduction}

It is estimated that the world needs a billion metric tons of wheat (Triticum aestivum L.) in the year 2024 compared to its current production level of 777 million metric tons (International Grains Council 2019). This extra demand is expected to be fulfilled mainly through conventional breeding. However, there is a growing concern among wheat breeders that the remaining variability in the bread wheat gene pool is insufficient to address current and future breeding objectives. It was therefore concluded that there is a need to broaden the genetic base of wheat (Hegde et al. 2002). The introduction of genes from Aegilops can contribute to obtaining favorable traits, including yield, quality, and resistance to biotic and abiotic stresses (Schneider et al. 2008). Aegilops species are also potential reservoirs of resistance genes to cold (Monneveux et al. 2000), salinity (Colmer

Communicated by E. Khlestkina.

Sylwia Okoń

sylwia.okon@up.lublin.pl

1 Institute of Plant Genetics, Breeding and Biotechnology, University of Life Sciences in Lublin, Akademicka 15, 20-950 Lublin, Poland et al. 2006), as well as heat and drought (Molnár et al. 2004; Zaharieva et al. 2001). Moreover, most Aegilops species have valuable resistance genes against pathogens (Schneider et al. 2008).

Genetic diversity analysis is an important tool for crop improvement programs. Accurate assessment of the levels of genetic diversity can be invaluable in crop breeding for identifying diverse parental plants to create segregating progenies with maximum genetic variability for further selection, as well as analyzing genetic variability in cultivars (Mohammadi and Prasanna 2003).

Retrotransposon-based marker methods such as interretrotransposon amplified polymorphism (IRAP) and retrotransposon-microsatellite amplified polymorphism (REMAP) are recent methods applied in genetic diversity analysis (Kalendar et al. 1999). REMAP is a method based on the amplification of DNA regions between retrotransposons and microsatellites using inter-simple sequence repeat (ISSR) primers and retrotransposon primers, while IRAP is based on the amplification of sequences between two retrotransposons using two retrotransposon primers (Roy et al. 2015).

The aim of this study was to assess the genetic diversity of 4 species belonging to the genus Aegilops, which are often used to expand wheat, as well as triticale genetic variability. 


\section{Materials and methods}

\section{Plant material}

The plant material consisted of 45 genotypes belonging to the genus Aegilops. All analyzed genotypes were hexaploid forms (based on data from the genebanks). Within the tested genotypes, 16 belonged to the species Aegilops neglecta Req. ex Bertol (6×), 5 to Aegilops vavilovii (Zhuk.) Chennav, 10 to Aegilops crassa Boiss. (6×) and 14 to Aegilops juvenalis (Thell.) Eig (van Slageren 1994; Bálint et al. 2000). Analyzed genotypes originated from two genebanks:

1. Institute of Plant Genetics and Crop Plant Research (IPK), Gatersleben, Germany (https://gbis.ipk-gaterslebe n.de/gbis2i/faces/index.jsf)

2. Germplasm Resources Information Network (GRIN), USDA, USA (https://npgsweb.ars-grin.gov/)

The genetic composition and origin of the studied genotypes are presented in Table 1.

\section{DNA isolation}

For DNA isolation, fragments of young seedlings of analyzed genotypes were collected and frozen in liquid nitrogen. Each sample consisted of plant material from 6 plants. DNA extraction was performed using the GeneMATRIX Plant \& Fungi DNA purification kit (EURx, Gdańsk, Poland) according to the manufacturer protocol. The isolated DNA was quantified and qualified using a NanoDrop (Thermo Scientific, Madison, USA) spectrophotometer.

\section{REMAP amplification}

In the first step, 23 microsatellite-specific primers and 2 REMAP primers were tested. Of the tested primers, 1 REMAP and 8 microsatellite primers were selected (Table 2).

\section{PCR amplification and visualization}

Polymerase chain reactions (PCRs) were performed in $20 \mu \mathrm{l}$ of a reaction mixture containing $40 \mathrm{ng}$ of genomic DNA, $1 \times$ Taq polymerase buffer (Fermentas), $2.5 \mathrm{mM} \mathrm{MgCl} 2$, $0.2 \mathrm{mM}$ of each dNTP (Fermentas), $0.4 \mu \mathrm{M}$ of each primer, and $1 \mathrm{U}$ of polymerase (Fermentas).

The PCR was performed using a Tprofessional Basic Gradient thermal cycler (Biometra). The PCR program was as follows: $95{ }^{\circ} \mathrm{C}$ for 3 min., 3 cycles: $95{ }^{\circ} \mathrm{C}$ for 30 s., $56{ }^{\circ} \mathrm{C}$ for 45 s., $72{ }^{\circ} \mathrm{C}$ for 1 min., 3 cycles: $95{ }^{\circ} \mathrm{C}$ for 30 s., $55^{\circ} \mathrm{C}$ for $45 \mathrm{~s} ., 72{ }^{\circ} \mathrm{C}$ for $1 \mathrm{~min} ., 32$ cycles: $95^{\circ} \mathrm{C}$ for 30 s., $54{ }^{\circ} \mathrm{C}$ for $45 \mathrm{~s} ., 72{ }^{\circ} \mathrm{C}$ for $1 \mathrm{~min}$. and a final extension at $72{ }^{\circ} \mathrm{C}$ for $10 \mathrm{~min}$.

The amplification products were electrophoretically separated in $1.5 \%$ agarose gel containing $0.01 \%$ ethidium bromide in $1 \times$ TBE buffer $(89 \mathrm{mM}$ Tris Base, $89 \mathrm{mM}$ boric acid, 2 mM EDTA pH 8.0). The separation was carried out for $3 \mathrm{~h}$ using $110 \mathrm{~V}$. The gel-separated DNA fragments were illuminated with a UV transilluminator and archived with the DigiGenius system (SynGene).

\section{Data analysis}

The bands were converted into a binary matrix where "1" was for the presence and " 0 " for the absence of a band at a particular position. Only bright and reproducible products were scored. The level of polymorphism of the primer (polymorphic products/total products) and relative frequency of polymorphic products (genotypes where polymorphic products were present/ total number of genotypes) (Belaj et al. 2001) were calculated. Resolving power of the primer was calculated using the formula: resolving power $(\mathrm{Rp})=\Sigma$ $\mathrm{Ib}$ (band informativeness). Band informativeness was calculated for each band scored by the primer individually. $\mathrm{Ib}=1-[2(0.5-\mathrm{p})], \mathrm{p}$ is the proportion of occurrence of bands in the genotypes out of the total number of genotypes (Prevost and Wilkinson 1999). Polymorphic information content (PIC) was calculated by applying the simplified formula (Anderson et al. 1993): PIC = 2fi(1-fi), where fi is the percentage of the ith amplified band present.

The genetic diversity parameters: percentage of polymorphic loci $(P \%)$, number of observed $(\mathrm{Na})$ and effective $(\mathrm{Ne})$ alleles, genetic diversity of $\mathrm{Nei}(\mathrm{He})$, Shannon index $(I)$ were calculated using GeneAIEx v.6.4 program (Peakall and Smouse 2012). To detect the distribution of genetic variation within and among populations, the analysis of molecular variance (AMOVA) and genetic distance parameters of Nei and $\mathrm{Li}$ (1979) were estimated. The main coordinate analysis (PCoA), using Euclidean distance, was performed to represent the genetic distances between the populations, through the GeneAIEx v.6.4 program (Peakall and Smouse 2012). The genetic similarities matrix from the calculative data was used to construct a dendrogram based on the unweighted pair group method with arithmetic averaging (UPGMA), and a dendrogram was constructed for all genotypes using PAST software (Hammer et al. 2001).

\section{Results}

Genetic diversity of 45 genotypes belonging to 4 species of the genus Aegilops was analyzed based on a set of 8 marker pairs, constituting a combination of the REMAP primer and 
Table 1 List of analyzed genotypes

\begin{tabular}{|c|c|c|c|c|c|}
\hline No. & Species & Genome* & Accession number & Genebank & Origin $* *$ \\
\hline 1 & \multirow[t]{16}{*}{ Aegilops neglecta Req. ex Bertol. (6×) } & \multirow[t]{16}{*}{ UMN } & AE 1440 & IPK & Greece \\
\hline 2 & & & AE 7 & IPK & Greece \\
\hline 3 & & & AE 169 & IPK & Portugal \\
\hline 4 & & & AE 301 & IPK & France \\
\hline 5 & & & AE 589 & IPK & Spain \\
\hline 6 & & & AE 303 & IPK & Italy \\
\hline 7 & & & AE 1017 & IPK & Bulgaria \\
\hline 8 & & & PI 374317 & GRIN & Montenegro \\
\hline 9 & & & PI 378191 & GRIN & Serbia \\
\hline 10 & & & PI 554259 & GRIN & Bosnia and Herzegovina \\
\hline 11 & & & PI 554262 & GRIN & Croatia \\
\hline 12 & & & PI 344776 & GRIN & Macedonia \\
\hline 13 & & & AE 129 & IPK & Russia \\
\hline 14 & & & AE 130 & IPK & Asia \\
\hline 15 & & & PI 276987 & GRIN & South Turkey \\
\hline 16 & & & PI 564203 & GRIN & Northwest Turkey \\
\hline 17 & \multirow[t]{5}{*}{ Aegilops vavilovii (Zhuk.) Chennav } & \multirow[t]{5}{*}{ DMS } & AE 30 & IPK & $($ Sweden $) * * *$ \\
\hline 18 & & & AE 1580 & IPK & $(\text { Morocco })^{* * *}$ \\
\hline 19 & & & AE 160 & IPK & Uzbekistan \\
\hline 20 & & & AE 363 & IPK & Turkmenistan \\
\hline 21 & & & AE 741 & IPK & Iran \\
\hline 22 & \multirow[t]{10}{*}{ Aegilops crassa Boiss. $(6 \times)$} & \multirow[t]{10}{*}{ DDM } & AE 898 & IPK & Tajikistan \\
\hline 23 & & & AE 815 & IPK & Turkmenistan \\
\hline 24 & & & PI 219958 & GRIN & South Afghanistan \\
\hline 25 & & & PI 317400 & GRIN & North Afghanistan \\
\hline 26 & & & PI 298892 & GRIN & Northeast Afghanistan \\
\hline 27 & & & PI 317393 & GRIN & Northwest Afghanistan \\
\hline 28 & & & PI 392330 & GRIN & Uzbekistan \\
\hline 29 & & & PI 276972 & GRIN & Uzbekistan \\
\hline 30 & & & PI 487286 & GRIN & Jordan \\
\hline 31 & & & PI 542178 & GRIN & West Turkey \\
\hline 32 & \multirow[t]{14}{*}{ Aegilops juvenalis (Thell.) Eig } & \multirow[t]{14}{*}{ DMU } & AE 91 & IPK & Great Britain \\
\hline 33 & & & AE 182 & IPK & Uzbekistan \\
\hline 34 & & & AE 497 & IPK & Uzbekistan \\
\hline 35 & & & PI 574463 & GRIN & Uzbekistan \\
\hline 36 & & & PI 542192 & GRIN & West Turkey \\
\hline 37 & & & AE 537 & IPK & No data \\
\hline 38 & & & AE 555 & IPK & No data \\
\hline 39 & & & AE 578 & IPK & No data \\
\hline 40 & & & AE 582 & IPK & No data \\
\hline 41 & & & AE 1495 & IPK & No data \\
\hline 42 & & & PI 266815 & GRIN & No data \\
\hline 43 & & & PI 276993 & GRIN & No data \\
\hline 44 & & & PI 289577 & GRIN & No data \\
\hline 45 & & & PI 330485 & GRIN & No data \\
\hline
\end{tabular}

*According to Kimber and Tsunewaki (1988)

**According to information provided by genebank

***Country of origin may be assumed as country of accession donor (accession Ae.vavilovii (Zhuk.) are not expected to originate from these regions) 
Table 2 The set of primers used for amplification

\begin{tabular}{ll}
\hline Primer name & Sequence $\left(5^{\prime}-3^{\prime}\right)$ \\
\hline ISSR 11 & $(\mathrm{AC})_{8} \mathrm{G}$ \\
ISSR 14 & $(\mathrm{GA})_{9} \mathrm{~A}$ \\
ISSR 16 & $(\mathrm{GA})_{8} \mathrm{C}$ \\
ISSR 17 & $(\mathrm{GA})_{8} \mathrm{YC} *$ \\
ISSR 23 & $(\mathrm{CA})_{8} \mathrm{GC}$ \\
ISSR 31 & $(\mathrm{AG})_{8} \mathrm{YC}$ \\
ISSR 32 & $(\mathrm{AG})_{8} \mathrm{YT}$ \\
ISSR 34 & $(\mathrm{CA})_{8} \mathrm{TG}$ \\
R-2 & $\mathrm{CTA} \mathrm{GGG} \mathrm{CAT}$ \\
& $\mathrm{AAT}$ TCC \\
& AAC A \\
\hline
\end{tabular}

*Y degenerate nucleotides

8 ISSR primers. In total, primers amplified 486 products for all analyzed forms, of which $96.09 \%$ were polymorphic. Primer pairs used for genotypes belonging to the species $A e$. neglecta amplified 218 products, 231 in Ae. crassa genotypes, 210 in Ae. vavilovii, and 195 in Ae. juvenalis. Among the obtained products, $174,175,138$, and 136 were polymorphic within the analyzed species, respectively.

The mean PIC value for the applied markers was 0.25 ; for individual species, it was 0.21 for Ae. neglecta, 0,23 for Ae. crassa and Ae. vavilovii, and 0.18 for Ae. juvenalis. The mean RP value was 28.13 , while for individual species, it was 26.31 (Ae. neglecta), 30.38 (Ae. crassa), 31,75 (Ae. vavilovii), and 27.32 (Ae. juvenalis). Detailed results obtained for particular primer pairs are presented in Table 3.

AMOVA was calculated as a measure of genetic diversity. The results indicated that $66 \%$ of the total variance occurred between species, while $34 \%$ was attributed to variation within the species. There was a significant genetic variation found $(P<0.001)$ between the analyzed Aegilops species. Detailed genetic variation parameters are presented in Table 4 . The percentage of polymorphic loci
Table 4 Distribution of the genetic diversity of 3 species of Aegilops L. based on REMAP markers

\begin{tabular}{llllll}
\hline Species & \multicolumn{5}{l}{ Genetic diversity parameters } \\
\cline { 2 - 6 } & $\mathrm{Na}$ & $\mathrm{Ne}$ & $I$ & $\mathrm{He}$ & $\% P$ \\
\hline Ae. neglecta & 0.807 & 1.141 & 0.140 & 0.089 & $35.80 \%$ \\
Ae. crassa & 0.835 & 1.169 & 0.157 & 0.101 & $36.01 \%$ \\
$\begin{array}{l}\text { Ae. carssa } \\
\quad 0.716\end{array}$ & 1.148 & 0.138 & 0.090 & $28.40 \%$ \\
$\quad$ vavsp. & & & & & \\
Ae. juvenalis & 0.681 & 1.115 & 0.112 & 0.071 & $27.98 \%$ \\
\hline
\end{tabular}

$(\% P)$ was 32.05 and ranged from 27.98 for Ae. juvenalis to 36.01 for Ae.crassa.

Ae. crassa showed the highest genetic diversity with $I=0.157$ and $\mathrm{He}=0.101$, while Ae. juvenalis showed the lowest one with $I=0.112$ and $\mathrm{He}=0.071$. Ae. crassa also showed the highest number of different bands and bands with a frequency $>=5 \%$. The highest number of private bands was also observed for this species (Fig. 1).

To explore the relationships among the individuals, the Dice genetic similarity coefficient was calculated based on the binary data matrix. The values of similarity indexes for all analyzed genotypes ranged from 0.210 to 0.987 , and the mean value was 0.466 . The similarity matrix index was also calculated for each species separately. The highest similarity values were obtained for genotypes belonging to the species Ae. juvenalis. They ranged from 0.612 to 0.987 , and the mean value was 0.826 . Similarity within Ae. neglecta was in the range of 0.510-0.978, and the mean value was 0.762 . The obtained results showed that genotypes belonging to Ae. crassa and Ae. vavilovii were the most diverse. The values of similarity coefficients for Ae. crassa were in the range of 0.517-0.969, with the mean value of 0.751 , while for Ae. vavilovii range of $0.625-0.964$, with the mean value of 0.748 .
Table 3 Characteristic of REMAP primers used to estimate genetic variability of the analyzed Aegilops L. genotypes

\begin{tabular}{lccccccc}
\hline Primer No & Total & Monomorphic & Polymorphic & $\begin{array}{l}\text { Specific for sin- } \\
\text { gle genotype }\end{array}$ & $\begin{array}{l}\text { Specific for } \\
\text { species }\end{array}$ & PIC & Rp \\
\hline $\begin{array}{l}\text { Number of bands } \\
\text { R-2- ISSR 11 }\end{array}$ & 61 & 2 & & & & \\
R-2- ISSR 14 & 36 & 5 & 59 & 16 & 5 & 0.24 & 27.87 \\
R-2-ISSR 16 & 67 & 5 & 31 & 3 & 3 & 0.27 & 26.31 \\
R-2-ISSR 17 & 73 & 1 & 62 & 11 & 5 & 0.23 & 34.18 \\
R-2- ISSR 23 & 81 & 1 & 71 & 16 & 5 & 0.24 & 24.27 \\
R-2 ISSR 31 & 52 & 2 & 80 & 22 & 3 & 0.19 & 21.51 \\
R-2- ISSR 32 & 74 & 1 & 50 & 5 & 3 & 0.33 & 37.64 \\
R-2- ISSR 34 & 42 & 1 & 73 & 19 & 5 & 0.23 & 29.82 \\
Total & 486 & 18 & 41 & 9 & 6 & 0.29 & 23.47 \\
\hline
\end{tabular}




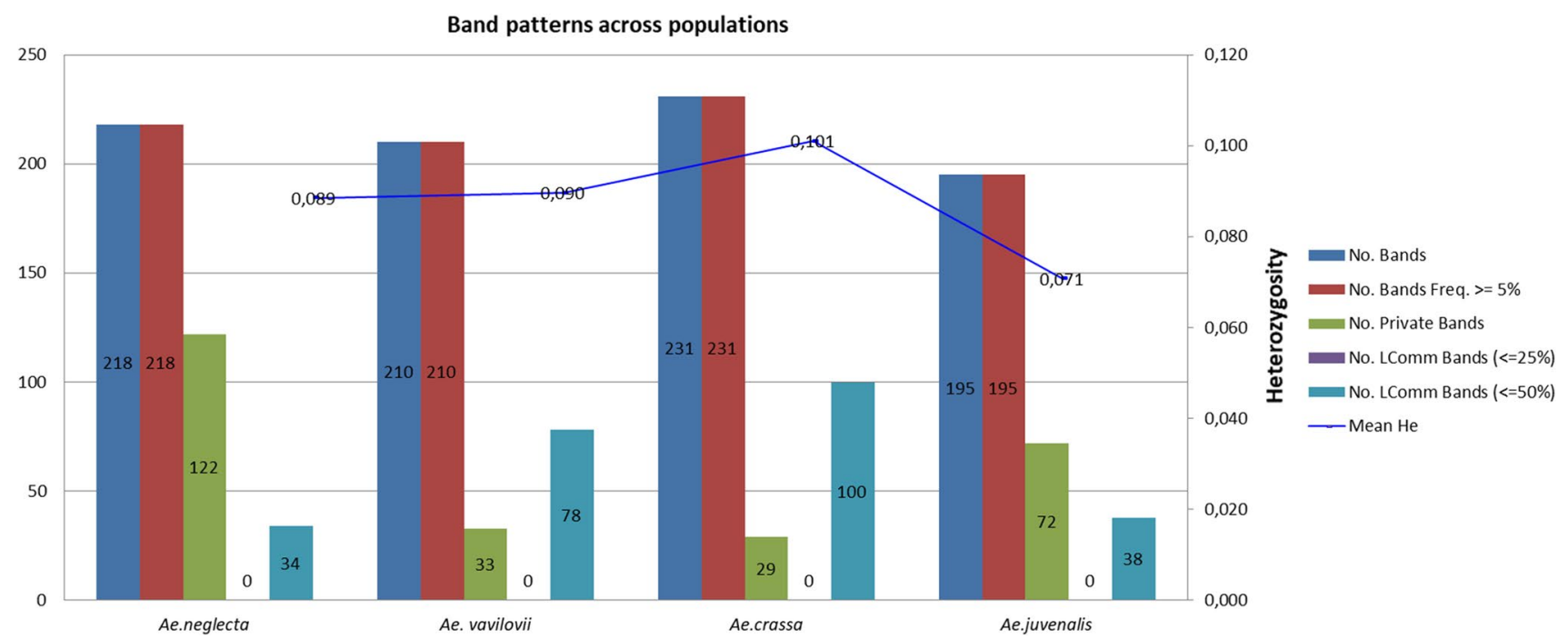

Fig. 1 Total bands patterns for the analyzed Aegilops species

The obtained similarity matrices of indexes were used to construct a dendrogram using the UPGMA method. Forty-five genotypes clustered into 3 separate groups. Genotypes belonging to Ae. neglecta and Ae. juvenalis formed separate groups, while Ae. crassa and Ae. vavilovii co-clustered in one group. On the dendrogram, Ae. crassa, Ae. vavilovii, and Ae. juvenalis genotype groups were located closer to each other. The group of Ae. neglecta genotypes formed a separate branch (Fig. 2).

The relationships between 45 analyzed genotypes were determined using PCoA. PCoA analysis led to the results comparable to those obtained using UPGMA clustering. Aegilops genotypes formed 3 distinct groups, corresponding to UPGMA clusters (Fig. 3). The first three principal components explained $67 \%$ of the total data variation,

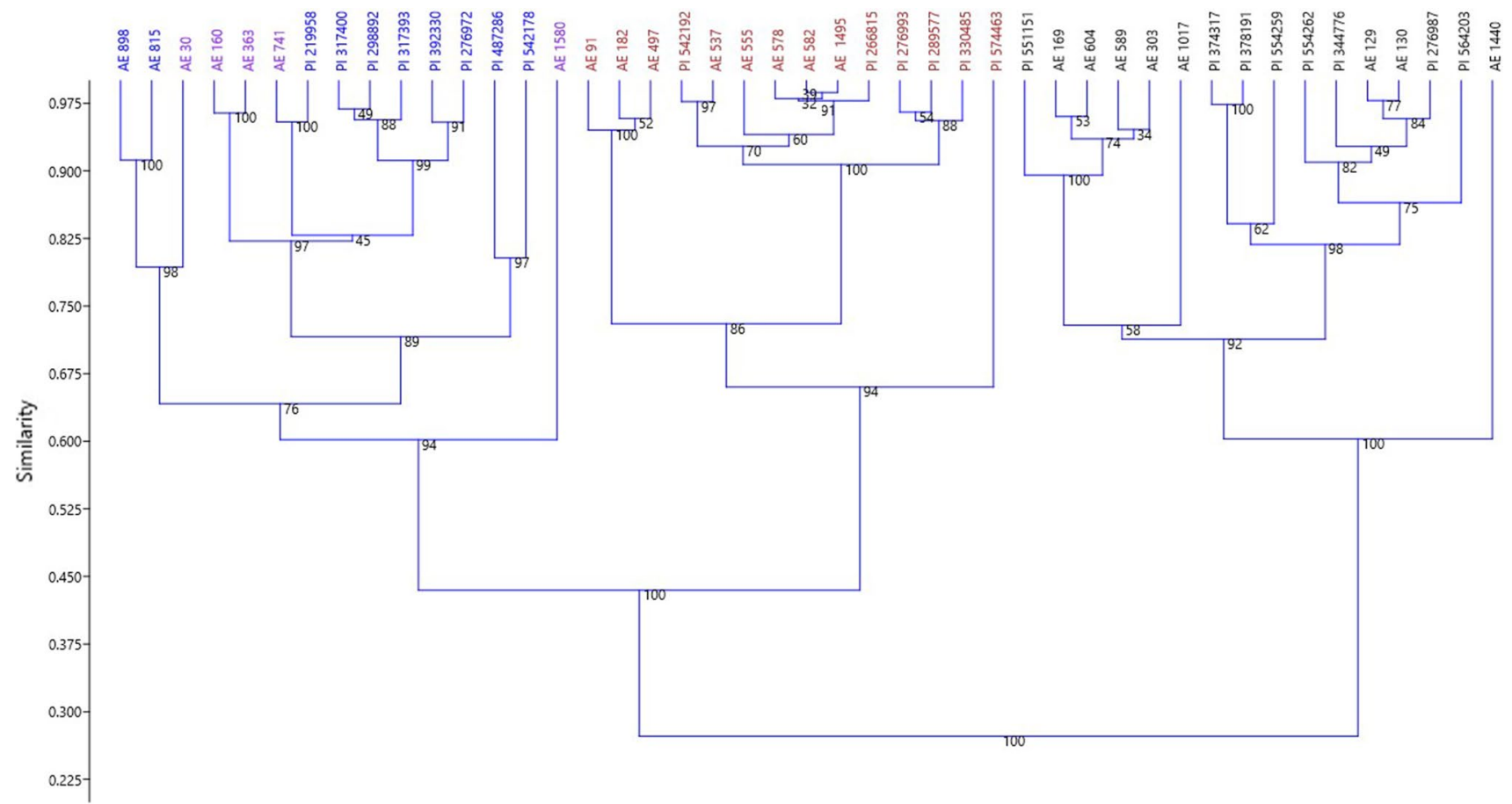

Fig. 2 Dendrogram of the 45 Aegilops accessions produced by Dice coefficient using UPGMA clustering method based on REMAP data 


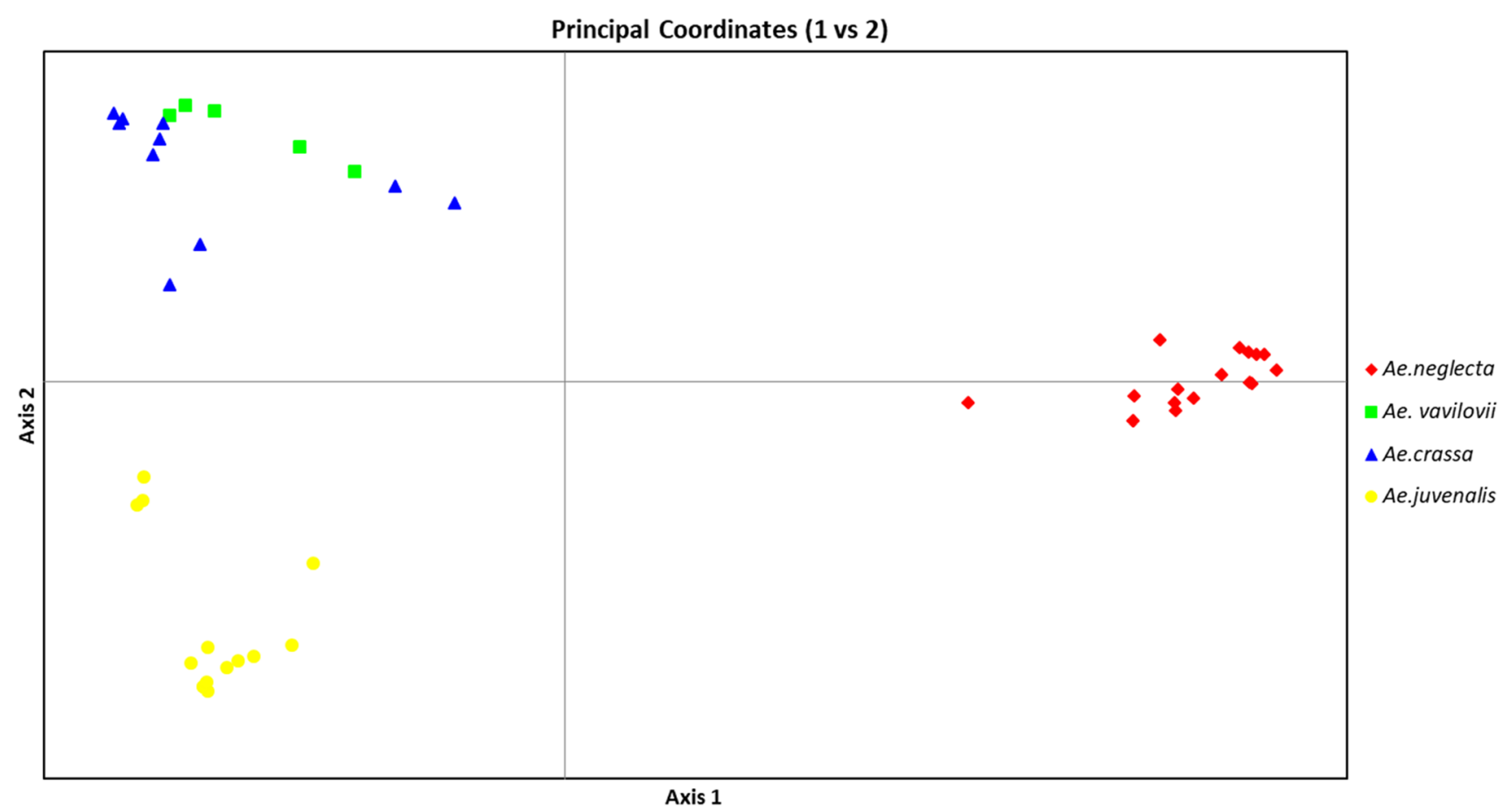

Fig. 3 Principal coordinate analysis of the Aegilops L. genotypes

while PC-1, PC2, and PC-3 accounted for 37.31, 24.21, and 5.48, respectively.

Separate dendrograms were created for each species based on the obtained matrices. Genotypes belonging to Ae. neglecta formed 2 groups of 6 and 9 genotypes, respectively. The first group contained 4 genotypes from Western Europe and 2 genotypes from Greece and Bulgaria. In the second group, genotypes from the Balkan countries and Asia and Turkey clustered together. The Ae1440 genotype from Greece was located on the outskirts of the dendrogram, and it differed the most from other genotypes belonging to the species Ae. neglecta.

Genotypes belonging to the species Ae. crassa formed 3 groups of genotypes with a similar origin. Genotypes from Afghanistan and Uzbekistan grouped together, similar to genotypes from Jordan and Turkey. In the third group genotypes from Tajikistan and Turkmenistan were clustered.

On the dendrogram constructed for Aegilops vavilovii genotypes, accessions from Uzbekistan and Turkmenistan formed a close group, while genotype from Morocco was most different and located on the outskirts of the dendrogram.

Ae. juvenalis genotypes formed two main cluster groups on the dendrogram. In group I, there were 3 subgroups with 2, 5, and 3 genotypes, respectively. Group II contained 3 genotypes. The PI574463 genotype was located on the periphery of the dendrogram.

\section{Discussion}

Molecular analysis using REMAP and ISSR markers is an efficient and inexpensive method to evaluate genetic diversity that has been used repeatedly to determine genetic relationships of many plants (Mahjoob et al. 2016; Ghufaili and Al-Tamimi 2017; Cheraghi et al. 2018; Vuorinen et al. 2018). REMAP markers generate a wide range of band patterns. They enable identification of polymorphisms between species as well as within a single species or cultivars (Kalendar et al. 1999; Branco et al. 2007; Mahjoob et al. 2016; Cheraghi et al. 2018).

In our study, REMAP markers were used to evaluate genetic diversity within and between 3 species of the genus Aegilops used in crossbreeding to introduce genetic diversity of triticale. Among the obtained bands, $96.09 \%$ were polymorphic, which has confirmed that these markers are an effective tool for the analysis of genetic diversity of species from the genus Aegilops. Paczos-Grzęda and Bednarek (2014) used REMAP markers to analyze polymorphism of hexaploid species of the genus Avena and obtained $73.5 \%$ of polymorphic products. Analogous studies on common wheat were conducted by Holasou et al. (2019), and these authors obtained the percentage of polymorphic loci (PPL) at the level of $86.4 \%$. REMAP markers were also used to analyze genetic similarity in 
triticale. The obtained level of polymorphic bands was 88.46\% (Kalinka and Achrem 2018).

PIC (polymorphism information content) and Rp (resolving power of the primer) are the measures of marker usefulness for similarity analyses (Powell et al. 1996). PIC is the probability of a primer detecting polymorphism between individuals and depends on the number of detectable alleles and distribution of their frequency. Rp specifies the discriminatory potential of primers and estimates the ability of the technique to produce optimally informative bands (Yousefi et al. 2015). In the current study, both PIC and Rp were calculated for the applied marker combinations. The obtained values ( $P I C=0.25$ and $R p=28.13$ ) confirmed the usefulness of the combination of REMAP and ISSR primers for the analysis of genetic similarity of genotypes belonging to the genus Aegilops. Taheri et al. (2018) analyzed genotypes of the genus Triticum and obtained a PIC of 0.40 using REMAP markers.

Genotypes belonging to different species of the genus Aegilops were analyzed in order to assess the level of genetic diversity. Pour-Aboughadareh et al. (2018) analyzed 8 Aegilops species and 4 Triticum species using SCoT (Start codon targeted) markers. Authors showed that SCoT markers, as well as REMAP markers, were a good tool for analyzing the genetic diversity of species within the genus Aegilops. They obtained $98.3 \%$ of polymorphic products. In our own research, the level of polymorphism was $96.09 \%$. Thomas and Bebeli (2010) also observed a high level of polymorphism Aegilops genotypes using the RAPD and ISSR method. Abbasov et al. (2019) used SSR markers to evaluate the genetic diversity of Aegilops genotypes from Azerbaijan and Georgia, but the number of obtained amplicons was much lower than for REMAP or SCoT markers. The results obtained in this study showed that the most diverse species were Ae. crassa and Ae. vavilovii. Genotypes belonging to these species exhibited the highest values of genetic variation parameters and the lowest values of similarity coefficients. Pour-Aboughadareh et al. (2018) demonstrated that Ae. cylindrica and Ae. umbellulata were characterized by the highest levels of genetic diversity among the analyzed Aegilops species, while Ae. crassa had a lower level of diversity. Research conducted by Pour-Aboughadareh et al. (2018) showed a lower level of Ae. crassa differentiation compared to Ae. neglecta. In the present study, Ae. crassa was characterized by the highest level of diversity, whereas Ae. neglecta had a medium level of similarity; Congruent conclusions can be found in the studies of Abbasov et al. (2019) and Pour-Aboughadareh et al. (2018). The authors of the latter study argued that such discrepancies in the results of many works could be caused by differences in the sample size or geographical origin of the analyzed accessions.

Clustering based on genetic distance was consistent with taxonomy. UPGMA and PCoA analyses based on REMAP markers allowed to discriminate all Aegilops species at the cluster level. No grouping according to the country within the species was observed, but we recorded a certain tendency of clustering the accessions from the same geographical regions. A similar trend was observed for genotypes from Azerbaijan and Georgia. In contrast, Pour-Aboughadared et al. (2018) showed that genetic diversity did not correspond to geographical distribution. They observed that all species clustered based on their genomic structure and these clusters were approximately consistent with their taxonomic classification. These differences may also result from a different number of samples and their different origin as well as the number of species analyzed.

In summary, we used molecular markers to investigate the variability within and between 4 hexaploid Aegilops species, which are often utilized in wheat breeding programs as a source of genetic diversity. Our research demonstrated that Ae. crassa and Ae. vavilovii were the most valuable species, which showed the highest values of genetic diversity parameters and the lowest genetic similarity indexes.

\section{Compliance with ethical standards}

Conflict of interest The authors declare that they have no conflict of interest.

Ethical approval This article does not contain any studies with human participants or animals performed by any of the authors.

Informed consent The authors agreed to the publication of the manuscript. All authors read and approved the final manuscript.

Open Access This article is licensed under a Creative Commons Attribution 4.0 International License, which permits use, sharing, adaptation, distribution and reproduction in any medium or format, as long as you give appropriate credit to the original author(s) and the source, provide a link to the Creative Commons licence, and indicate if changes were made. The images or other third party material in this article are included in the article's Creative Commons licence, unless indicated otherwise in a credit line to the material. If material is not included in the article's Creative Commons licence and your intended use is not permitted by statutory regulation or exceeds the permitted use, you will need to obtain permission directly from the copyright holder. To view a copy of this licence, visit http://creativecommons.org/licenses/by/4.0/.

\section{References}

Abbasi Holasou H, Rahmati F, Rahmani F, Imani M, Talebzadeh Z (2019) Elucidate genetic diversity and population structure of bread wheat (Triticum Aestivum L.) cultivars using IRAP and REMAP markers. J Crop Sci Biotechnol 22:139-151

Abbasov M et al (2019) Genetic diversity of Aegilops L. species from Azerbaijan and Georgia using SSR markers. Genet Resour Crop Evol 66:453-463 
Anderson JA, Churchill GA, Autrique JE, Tanksley SD, Sorrells ME (1993) Optimizing parental selection for genetic linkage maps. Genome 36:181-186

Bálint AF, Kovács G, Sutka J (2000) Origin and taxonomy of wheat in the light of recent research. Acta Agron Hung 48(3):301-313

Belaj A, Trujillo I, De La Rosa R, Rallo L, Giménez MJ (2001) Polymorphism and discrimination capacity of randomly amplified polymorphic markers in an olive germplasm bank. J Am Soc Hort Sci 126:64-7164

Branco CJS et al (2007) IRAP and REMAP assessments of genetic similarity in rice. J Appl Genet 48:107-113

Cheraghi A, Rahmani F, Hassanzadeh-Ghorttapeh A (2018) IRAP and REMAP based genetic diversity among varieties of Lallemantia iberica. Mol Biol Res Commun 7:125-132

Colmer TD, Flowers TJ, Munns R (2006) Use of wild relatives to improve salt tolerance in wheat. J Exp Bot 57:1059-1078

Ghufaili MF, Al-Tamimi AJT (2017) Genetic relationship among some wheat genotypes using ten ISSR markers. IOSR J Pharm Biol Sci $12: 30-36$

Hammer $\varnothing$, Harper DAT, Ryan PD (2001) Past: paleontological statistics software package for education and data analysis. Palaeontol Electronica 4:5-7

Hegde SG, Valkoun J, Waines JG (2002) Genetic diversity in wild and weedy Aegilops, Amblyopyrum, and Secale species-a preliminary survey. Crop Sci 42:608-614

International Grains Council (2019) Five-year baseline projections of supply and demand for wheat, maize (corn), rice and soyabeans to $2023 / 24$

Kalendar R, Grob T, Regina M, Suoniemi A, Schulman A (1999) IRAP and REMAP: two new retrotransposon-based DNA fingerprinting techniques. Theor Appl Genet 98:704-711

Kalinka A, Achrem M (2018) Reorganization of wheat and rye genomes in octoploid triticale $(\times$ Triticosecale). Planta 247:807-829

Kimber G, Tsunewaki K (1988) Genome symbols and plasma types in the wheat group. In: Proceedings of the 7th International Wheat Genetics Symposium, pp 1209-1210

Mahjoob B, Zarini HN, Hashemi SH, Shamasbi FV (2016) Comparison of ISSR, IRAP and REMAP markers for assessing genetic diversity in different species of Brassica sp. Rus J Genet 52:1272-1281

Mohammadi SA, Prasanna BM (2003) Analysis of genetic diversity in crop plants-salient statistical tools and considerations. Crop Sci 43:1235

Molnár I et al (2004) Physiological and morphological responses to water stress in Aegilops biuncialis and Triticum aestivum genotypes with differing tolerance to drought. Funct Plant Biol 31:1149

Monneveux P, Zaharieva M, Rekika D (2000) The utilisation of Triticum and Aegilops species for the improvement of durum wheat, accessed 9 Sep 2019
Nei M, Li W-H (1979) Mathematical model for studying genetic variation in terms of restriction endonucleases. In: Proceedings of the National Academy of Sciences of the United States of America 76

Paczos-Grzeda E, Bednarek PT (2014) Comparative analysis of hexaploid Avena species using REMAP and ISSR methods. Turk J Bot 38:1103-1111

Peakall R, Smouse PE (2012) GenAlEx 6.5: genetic analysis in excel. population genetic software for teaching and research-an update. Bioinformatics 28:2537-2539

Pour-Aboughadareh A, Ahmadi J, Mehrabi AA, Etminan A, Moghaddam M (2018) Insight into the genetic variability analysis and relationships among some Aegilops and Triticum species, as genome progenitors of bread wheat, using SCoT markers. Plant Biosyst 152:694-703

Powell W et al (1996) The comparison of RFLP, RAPD, AFLP and SSR (microsatellite) markers for germplasm analysis. Mol Breed 2:225-238

Prevost A, Wilkinson MJ (1999) A new system of comparing PCR primers applied to ISSR fingerprinting of potato cultivars. Theor Appl Genet 98:107-112

Roy NS, Choi J-Y, Lee S-I, Kim N-S (2015) Marker utility of transposable elements for plant genetics, breeding, and ecology: a review. Genes Genom 37:141-151

Schneider A, Molnár I, Molnár-Láng M (2008) Utilisation of Aegilops (goatgrass) species to widen the genetic diversity of cultivated wheat. Euphytica 163:1-19

Taheri MT, Alavi-Kia SS, Mohammadi SA, Vahed MM (2018) Assessment of genetic diversity and relationships among Triticum urartu and Triticum boeoticum populations from Iran using IRAP and REMAP markers. Genet Resour Crop Evol 65:1867-1878

Thomas KG, Bebeli PJ (2010) Genetic diversity of greek Aegilops species using different types of nuclear genome markers. Mol Phylogenet Evol 56:951-961

Vuorinen A et al (2018) Retrotransposon-based genetic diversity assessment in wild emmer wheat (Triticum turgidum ssp. dicoccoides). Agronomy 8:107

van Slageren MW, (1994) Wild wheats: a monograph of Aegilops L. and Amblyopyrum (Jaub. \& Spach) Eig. Wageningen Agricultural University Papers 1994(7)

Yousefi V, Najaphy A, Zebarjadi A, Safari H (2015) Molecular characterization of thymus species using ISSR markers. J Animal Plant Sci 25:1087-1094

Zaharieva M, Gaulin E, Havaux M, Acevedo E, Monneveux P (2001) Drought and heat responses in the wild wheat relative aegilops geniculata roth: potential interest for wheat improvement. Crop Science Society of America 